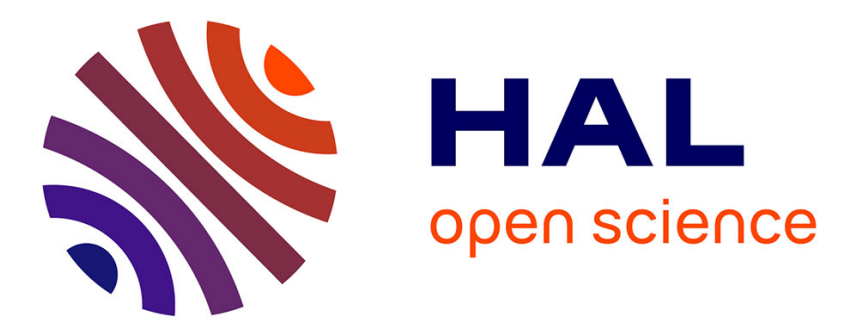

\title{
Les classes moyennes en Côte d'Ivoire. Des réalités contrastées
}

\author{
Anne Bekelynck, Jean-Philippe Berrou, Christian Bouquet, Dominique \\ Darbon
}

\section{- To cite this version:}

Anne Bekelynck, Jean-Philippe Berrou, Christian Bouquet, Dominique Darbon. Les classes moyennes en Côte d'Ivoire. Des réalités contrastées. Afrique Contemporaine, 2017, Côte d'Ivoire, le retour de l'éléphant ?, 263-264, pp.226-229. 10.3917/afco.263.0226 hal-02280172

\section{HAL Id: hal-02280172 \\ https://hal.science/hal-02280172}

Submitted on 6 Sep 2019

HAL is a multi-disciplinary open access archive for the deposit and dissemination of scientific research documents, whether they are published or not. The documents may come from teaching and research institutions in France or abroad, or from public or private research centers.
L'archive ouverte pluridisciplinaire HAL, est destinée au dépôt et à la diffusion de documents scientifiques de niveau recherche, publiés ou non, émanant des établissements d'enseignement et de recherche français ou étrangers, des laboratoires publics ou privés. 


\title{
Les classes moyennes en Côte d'Ivoire : Des réalités contrastées
}

\author{
Anne Bekelynck, Jean-Philippe Berrou, Christian Bouquet, \\ Dominique Darbon
}

\begin{abstract}
Jean-Philippe Berrou, Christian Bouquet et Dominique Darbon sont chercheurs au LAM (CNRS) et Sciences Po (Bordeaux). Anne Bekelynck est chercheuse au PAC-CI, ANRS (Côte d'Ivoire).

L'expansion des classes moyennes dans les pays en développement et émergents est présentée comme l'un des phénomènes majeurs de ce début de $\mathrm{XXI}^{\mathrm{e}}$ siècle. Les acteurs internationaux du développement estiment notamment que les classes moyennes pourraient avoir des effets d'entraînement au plan socio-économique dans ces pays et au-delà : expansion de la consommation et des marchés, soutien à la croissance, transformation des systèmes productifs, etc. Aujourd'hui, cette question est devenue un enjeu de controverses scientifiques majeur. Le cas de la Côte d'Ivoire en est une bonne illustration.
\end{abstract}

Le phénomène des classes moyennes peut s'étudier selon différentes approches ${ }^{1}$. L'approche économique utilise principalement un critère de revenu, de consommation, voire de richesse, afin de différencier les ménages ou individus de la classe moyenne de ceux considérés comme pauvres et comme riches. D'autres approches, plus sociologiques et politiques, se fondent sur l'observation du statut social (éducation, catégorie socioprofessionnelle, type d'emploi) et sur l'observation du prestige et/ou de l'identité de classe (préférences, aspirations). En marketing, ce sont les habitudes de consommation et leurs transformations qui sont observées. Il résulte de cette grande diversité d'approches un usage souvent peu précautionneux et parfois confus de l'expression «classe moyenne ». Cela n'a toutefois pas empêché son utilisation et sa diffusion massive par différents acteurs du développement global (banques régionales de développement, banques privées, cabinets de consultance). En Afrique, l'engouement pour les classes moyennes

\footnotetext{
${ }^{1} \mathrm{Ce}$ « Repère » est issu d'un projet de recherche comparatif financé par l'Agence française de développement (AFD) portant sur « Les implications de l'essor des classes moyennes dans les pays en développement et émergents », portant sur quatre pays distincts : le Brésil, la Côte d'Ivoire, la Turquie et le Vietnam (Berrou et al., 2018a, 2018b). Nous remercions l'Institut national de la statistique de Côte d'Ivoire pour la mise à disposition des données de l'enquête sur les conditions de vie des ménages de 2015 (ENV 2015) et l'ENSEA pour sa participation à la conduite et à l'analyse des entretiens semi-directifs.
} 
a largement été porté par un rapport de la Banque africaine de développement (BAD) publié en 2011, estimant que près d'un tiers de la population du continent appartenait à la classe moyenne (AfDB, 2011). Les magazines économiques et les cabinets internationaux de marketing lui ont emboîté le pas. Dans les faits, pourtant, les changements semblent beaucoup moins nets et font désormais douter ouvertement de la pertinence de l'usage de l'expression «classe moyenne » dans ces contextes (Melber, 2016 ; Kroeker et al., 2017). La Côte d'Ivoire en est une bonne illustration. Si la notion de classe moyenne est quasi absente des documents officiels (elle n'apparaît qu'une seule fois dans le Plan national de développement 2016-2020), un buzz médiatique est entretenu à grands frais par les acteurs économiques des marchés de la consommation et de la publicité dans le pays. En témoignent deux récentes études de marché sur la classe moyenne abidjanaise, conduites par IPSOS en 2015 et en 2017 pour le compte de la CFAO et d'Unilever (CFAO, 2015 ; UCT-Unilever, 2017).

\section{Le long déclin des classes moyennes ivoiriennes}

Évoquer aujourd'hui le réveil des classes moyennes en Côte d'Ivoire prend un sens particulier. Chacun a en effet en mémoire les « vingt glorieuses » des années 1960 à 1980, cette période du « modèle » ou du « miracle » ivoirien qui avait permis à Félix Houphouët- Boigny de narguer son voisin ghanéen Kwame Nkrumah en lui démontrant qu'il avait eu tort de choisir le « modèle communiste ». À cette époque, grâce à la fameuse Caisse de stabilisation, des dizaines de milliers de planteurs, de la filière cacao notamment, appartenaient à la classe moyenne, ainsi que bon nombre de fonctionnaires bien calés derrière des rémunérations avantageuses, et des salariés d'un secteur privé florissant, en particulier à Abidjan. La machine s'est ensuite enrayée au début des années 1980. D'abord sous l'effet des premiers programmes d'ajustement structurel (PAS) qui eurent pour conséquence principale un sérieux tour de vis sur les salaires de la fonction publique, malgré le combat d'arrière-garde du président Houphouët-Boigny pour « décrocher » les enseignants, les magistrats et les militaires de la grille salariale imposée par les institutions de Bretton Woods. Puis le père de l'indépendance, ancien fondateur du puissant syndicat des planteurs, perdit la « guerre du cacao », avec à la clé une division par deux du prix garanti aux producteurs. Enfin, la dévaluation du franc CFA en janvier 1994 - juste après sa mort en décembre 1993 car il s'y était toujours opposé - et la période qui suivit achevèrent de « déclasser » ceux qui avaient commencé à goûter à l'aisance et contribuèrent à une « séparation irréversible » entre l'élite dominante et le reste de la population. 
Comme souvent, l'instabilité politique accompagna cette évolution. Celle-ci commença avec la succession d'Houphouët, disputée entre Henri Konan Bédié et Alassane Ouattara, rapidement envenimée par des tensions ethnonationalistes autour de «l'ivoirité ». La Côte d'Ivoire connut alors son premier coup d'État militaire fin 1999 et fut aspirée dans une spirale de turbulences que le régime de Laurent Gbagbo (2000-2011) ne parvint pas à juguler. Dans le même temps, et même si les fondamentaux de l'économie ivoirienne ne s'effondrèrent pas, les taux de croissance jusque-là prometteurs finirent par passer dans le rouge. Autant dire que la classe moyenne n'existait pratiquement plus à la sortie de la crise postélectorale, en avril 2011. Depuis, le pays s'est redressé et a retrouvé un rythme moyen de croissance du PIB autour de 8 à $9 \%$ par an, grâce à des investissements ambitieux, à un soutien sans faille de la communauté internationale et au retour des gros opérateurs étrangers. Cette croissance a contribué à booster le pouvoir d'achat et la consommation des ménages, ce qui pourrait laisser penser que la classe moyenne est en cours de reconstitution. Afin d'analyser ce phénomène, nous avons mené une recherche pour tenter d'identifier et de caractériser les classes moyennes ivoiriennes contemporaines. Pour ce faire, nous avons utilisé une méthodologie originale qui combine de manière séquentielle des méthodes quantitatives et qualitatives. Les données mobilisées sont issues de l'Enquête niveau de vie (ENV) des ménages ivoiriens, menée en 2015 par l'Institut national de la statistique (INS) et portant sur un échantillon représentatif d'environ 13000 ménages, ainsi que d'une trentaine d'entretiens semi-directifs menés à Abidjan et Soubré. Notre méthodologie combine une approche économique basée sur le revenu et une approche plus sociologique et multidimensionnelle mobilisant des informations sur les conditions de vie (en particulier l'emploi et l'éducation).

\section{Une « classe moyenne » limitée à 26,4\% de la population en 2015}

Nous avons appréhendé la catégorie «gens du milieu » sur la base des revenus. On estime que la classe moyenne en Côte d'Ivoire se situe entre une borne inférieure absolue fixée à 4 dollars par jour et par tête en parité de pouvoir d'achat (soit un niveau deux fois supérieur au seuil de pauvreté de 2 dollars) et une borne supérieure relative qui exclut les $5 \%$ les plus riches (le percentile 95). La taille moyenne des ménages de cette classe étant de trois personnes, on obtient un revenu mensuel moyen par ménage approximativement compris entre 85200 francs CFA 
(360 dollars) et 405000 francs CFA (1 710 dollars) ${ }^{2}$. Cela concerne donc une large gamme de revenus, mais cette fourchette ne représente qu'une proportion relativement limitée de la population ivoirienne, soit $26,4 \%^{3}$. Cette «classe moyenne» ivoirienne présente quelques caractéristiques globales intéressantes. D'abord, les chefs de ménage de cette catégorie sont analphabètes à 48,1\%, ce qui apparaît en décalage avec les représentations habituelles. Ensuite, si $60 \%$ habitent en ville, seuls $16 \%$ sont Abidjanais. Ils affichent des comportements communs, notamment la même importance accordée à l'éducation des enfants, ce qui conduit la plupart de ces familles à opter pour l'enseignement privé, et donc à consacrer à ce poste budgétaire une part importante des revenus, passant souvent par un effort d'épargne conséquent.

\section{Un « monde du milieu » très hétéroclite : les milieux du milieu.}

Reste alors à étudier la cohérence de cet ensemble constituant un quart de la population du pays. Force est de constater que l'on passe d'une catégorie limitée de «gens du milieu » à une catégorie éclatée de «gens de milieux différents». Ainsi, sur la base de cinq variables caractérisant l'emploi et l'éducation du chef de ménage (CSP, statut dans l'emploi, secteur institutionnel, pluriactivité et niveau d'éducation), cinq grands groupes ont pu être identifiés. La classe moyenne des travailleurs de l'informel constitue la part la plus importante (39\% du total). Les chefs de ménage y sont plutôt jeunes et leur famille est plus petite. Pour la plupart, ils sont entrepreneurs indépendants dans le secteur des transports routiers, du commerce et de la petite industrie manufacturière non déclarée. Une part significative de leurs revenus, qui sont plutôt faibles, est dédiée aux transferts (redistribution à la famille élargie). Beaucoup aspirent à devenir de véritables entrepreneurs. La classe moyenne des agriculteurs - et en particulier des planteurs du sud- ouest - représente $25 \%$ du total. Naturellement installés en milieu rural, ils sont relativement âgés et, s'ils attachent beaucoup d'importance à la scolarisation de leurs enfants, leur faible revenu ne leur permet pas d'y consacrer beaucoup d'argent. Ils apparaissent

\footnotetext{
${ }^{2}$ Les conversions dollars-francs CFA sont en parité de pouvoir d'achat - PPA (le facteur de conversion de 2015 est de 236,641; source : Banque mondiale). Notons par ailleurs que la « taille ménages » estimée dans l'ENV 2015 est particulièrement faible en comparaison du recensement de la population.

${ }^{3}$ Cette proportion est à mettre en regard des estimations proposées par la BAD et certains de ces auteurs avec une classe moyenne ivoirienne évaluée en 2010, selon leur propre définition, alternativement à $37 \%$ de la population (BAD, 2011) ou $55 \%$ selon Ncumbe et Lufumpa (2015). Cette proportion de 26,4\% est aussi limitée au regard des autres pays de notre étude et notamment du Vietnam où avec la même définition (4 dollars-P95) la classe moyenne vietnamienne est estimée à 72,5\% de la population en 2012.
} 
très vulnérables et se plaignent notamment d'être à la merci des aléas climatiques et des fluctuations du marché sur les produits d'exportation (café, cacao, hévéa, etc.). Ils « vivent selon la courbe ». La classe moyenne des retraités et inactifs (15\%), souvent représentée par des femmes, apparaît comme vulnérable et dépendante des transferts familiaux. Elle se singularise par le fait que ses membres sont souvent propriétaires de leurs logements. Mais leurs revenus sont en moyenne les plus bas de cette classe moyenne et l'augmentation de l'espérance de vie risque arithmétiquement de dégrader leur condition. Les dirigeants, cadres et professions intermédiaires du secteur public constituent le troisième sous-groupe (17\%). Ils affichent les plus hauts niveaux moyens de revenu et d'éducation, et appartiennent pour beaucoup d'entre eux au secteur de l'éducation et de l'enseignement supérieur où les salaires sont relativement élevés. Mais une part substantielle de leurs revenus peut provenir d'une ou de plusieurs autres activités, notamment en tant «qu'agriculteurs absentéistes». Enfin, la classe moyenne intermédiaire du secteur privé formel (4\%) rassemble des employés et salariés bien rémunérés, représentés surtout dans les secteurs de l'immobilier, du commercial et de la finance. Ils sont davantage que les autres connectés à Internet et dépensent beaucoup dans l'éducation des enfants, la santé et les communications. Eux aussi investissent volontiers dans l'immobilier ou les plantations.

\section{«Classe moyenne » : une expression performative}

Au-delà de ces cinq groupes, un fort dualisme se dégage. D'un côté, on trouve en minorité (21\%) une strate haute et stabilisée de la classe moyenne, constituée vraisemblablement plutôt d'héritiers des groupes intermédiaires des années 19601970. De l'autre, on observe une strate basse majoritaire $(79 \%)$ et instable, composée de ménages en situation de petite prospérité en même temps que de grande vulnérabilité et dont les trajectoires sociales ascendantes et descendantes alternent au gré des différents chocs (économiques, sociaux, politiques, climatiques, etc.) auxquels ces populations sont confrontées. Ainsi, ce qui, dans un pays comme la Côte d'Ivoire, est qualifié d'émergence des classes moyennes correspond en fait à deux réalités différentes : pour l'essentiel à un enrichissement régulier des catégories les plus aisées, la notion de «classe moyenne » caractérisant alors les ménages les plus riches de cette catégorie et des trajectoires de sortie de pauvreté, instables et variables dans leur durée et leur fréquence, sans toutefois que ces dynamiques soient suffisantes pour accroître et stabiliser à court terme la base de cette classe moyenne et confirmer son existence de manière durable. Le plus souvent notre population (4 dollars-P95) se définira par le «ni-ni », ni riche, ni pauvre, signifiant non 
pas qu'on se trouve au milieu, mais un rejet total de la pauvreté et des pauvres dont on vient et une attraction pour ce qui est impossible pour l'instant, la catégorie des riches. Pour autant, ce « ni-ni » ne permet pas de constituer une catégorie en soi et pour soi. En dépit de son ancienneté et en partie en raison de la crise économique et politique des années 19802000, la catégorie moyenne en Côte d'Ivoire n'a pu se stabiliser autour d'un noyau dur d'héritiers stabilisant des manières de faire. Les résultats de nos «entretiens- ménages » montrent d'ailleurs que ces classes moyennes sont essentiellement mues par la recherche d'intérêts personnels et assez matériels (conditions de vie, logement, sécurité, etc.) et par la protection de leurs stratégies de promotion personnelle et familiale. Dans ce cadre, et compte tenu de la forte hétérogénéité de cette catégorie, les mobilisations collectives sont très limitées et l'altruisme politique réduit. L'expression politique, lorsqu'elle existe, repose davantage sur l'identité socioprofessionnelle (fonctionnaires notamment) que sur l'appartenance à une quelconque classe moyenne. Que nous révèle finalement notre étude sur les classes moyennes en Côte d'Ivoire ? D'une part, la confirmation de l'existence d'une Upper Middle Class minoritaire (21\%), dont le mode de vie se rapproche des niveaux de la Global Middle Class internationale. D'autre part, l'identification de mouvements de sortie de pauvreté hésitants, fragiles et à la marge laissant toutefois ouvert l'espoir d'une transformation tendancielle de la structure sociale du pays. Enfin, le tout est recouvert d'un discours performatif sur la «classe moyenne», investi désormais par les politiques, les investisseurs et les bailleurs comme une sorte de «multiplicateur d'investissement sociologique ».

\section{Bibliographie}

African Development Bank (2011), "Dynamics of the Middle Class in Africa", Market Brief. Berrou, J.-P., Clément, M., Combarnous, F., Darbon, D., Rougier, E. (2018a), «L'essor des classes moyennes dans les pays en développement et émergents : une étude comparative des enjeux d'identification, de caractérisation et de politiques publiques. Brésil, Côte d'Ivoire, Turquie, Vietnam », « Papiers de Recherche », Paris, AFD

Berrou, J.-P., Darbon, D., Bekelynck, A., Bouquet, C., Clément, M., Combarnous, F., Rougier, E. (2018b), «Le réveil des classes moyennes ivoiriennes ? Identification, caractérisation et implications pour les politiques publiques », «Papiers de Recherche », Paris, AFD.

CFAO (2015), «Les classes moyennes en Afrique, quelle réalité ? quels enjeux ? », White Paper, CFAOIPSOS- Bearing Point. Kroeker, L., O’Kane, D., Scharrer, T. (2017), Middle 
Classes in Africa. Changing Lives and Conceptual Challenges, Londres, Palgrave Macmillan.

Melber, H. (2016), The Rise of Africa's Middle Class, Londres, Zed Books.

Ncube, M., Lufumpa, C.L. (2015), The Emerging Middle Class in Africa, Londres, Routledge.

UCT-Unilever Institute of Strategic Marketing (2017), “The African Middle Class. Who are they?", rapport. 